\title{
A Reconfigurable Wideband and Multiband Antenna Using Dual-Patch Elements for Compact Wireless Devices
}

\author{
Hattan F. Abutarboush, R. Nilavalan, S. W. Cheung, K. M. Nasr, T. Peter, D. Budimir and H. Al-Raweshidy
}

\begin{abstract}
A reconfigurable wideband and multiband C-Slot patch antenna with dual-patch elements is proposed and studied. It occupies a compact volume of $50 \times 50 \times 1.57\left(3925 \mathrm{~mm}^{3}\right)$, including the ground plane. The antenna can operate in two dual-band modes and a wideband mode from 5 to $7 \mathrm{GHz}$. Two parallel C-Slots on the patch elements are employed to perturb the surface current paths for excitation of the dual-band and the wideband modes. Two switches, implemented using PIN diodes, are placed on the connecting lines of a simple feed network to the patch elements. Dual-band modes are achieved by switching "ON" either one of the two patch elements, while the wideband mode with an impedance bandwidth of $33.52 \%$ is obtained by switching "ON" both patch elements. The frequencies in the dual-band modes can be independently controlled using positions and dimensions of the C-Slots without affecting the wideband mode. The advantage of the proposed antenna is that two dual-band operations and one wideband operation can be achieved using the same dimensions. This overcomes the need for increasing the surface area normally incurred when designing wideband patch antennas. Simulation results are validated experimentally through prototypes. The measured radiation patterns and peak gains show stable responses and are in good agreements. Coupling between the two patch elements plays a major role for achieving the wide bandwidth and the effects of mutual coupling between the patch elements are also studied.
\end{abstract}

Index Terms - Patch Antenna, Wideband Antenna, Reconfigurable Antenna, Switched Antenna, Small Antenna, Slot Antenna, C-Slot

\section{INTRODUCTION}

$\mathrm{P}$ ATCH antennas suffer from narrow bandwidth which can limit their uses in some modern wireless applications [1] [2], therefore there is an increasing demand for low profile, easy to manufacture and multiband/wideband antennas which can be easily integrated within communication systems. A variety of studies have come up with different techniques to

Manuscript received January 09, 2011.

(Hattan F. Abutarboush, R. Nilavalan and T. Peter are with the Wireless Networks and Communications Centre (WNCC), School of Engineering and Design, Brunel University, West London, UB8 3PH.UK. e-mail: (Hattan.Abutarboush@ieee.org).

S. W. Cheung is with the department of Electrical and Electronics Engineering, Hong Kong University, Hong Kong.

K. Nasr is with the National Physical Laboratory (NPL), Teddington, TW11 OLW, UK.

D. Budimir is with the Wireless Communications Research Group, Department of Electronics and Computer Science, Westminster University, W1W 6UW, London, UK. achieve wideband operation for printed antennas. Some of the techniques employed are changing the physical size of the antenna, modifying the radiator shape to allow current paths to travel at longer distances (which sometime increases the antenna size), and adding additional parts such as multi layers or gaps (which again makes the antenna larger and of a higher profile). Other techniques include using U-slot array [3], shorting wall [4], folded shorting wall [5], Y-V Slot [6], slots form [7], stacked patch [8], pair of slits on the patch (with total size of the antenna $150 \times 150 \times 14.3 \mathrm{~mm}^{2}$ ) [9], E-shaped patch on thick substrates with ground plane size of $140 \times 210 \mathrm{~mm}^{2}$ [10] and using circular arc shaped slot on thick substrate [11]. The designs in [3]-[11] can achieve wide impedance bandwidths. However, these antennas are large in sizes and difficult to fit into small and slim devices.

A reconfigurable antenna is another solution to achieve a wide impedance bandwidth by switching $\mathrm{ON}$ and OFF some parts of the antenna. To allow the operating frequencies and the bandwidths to be reconfigurable, switching components are normally used. PIN diodes, varactor diodes or MEMS switches are the most frequently used components in the design of reconfigurable antennas [12]-[15]. In literature, few papers reported the approach of switching between wideband and narrowband operations. For example in [16], studies were done on switching between dual ports, one port for Ultra Wideband (UWB) and the other port for a single narrowband. In [17]-[18], switching between UWB and a single narrowband was reported. However, the use of an UWB antenna for multiband applications could result in unwanted emissions in the transmission mode.

In this paper, a single-feed reconfigurable wideband and multiband antenna using two patch elements on a planar structure is proposed. Two C-Slots are employed on the patch elements for excitation of the dual-band and wideband modes. Two PIN diode switches are placed on the connecting lines of a simple feed network to the patch elements. Dual-band and wideband-mode operations are obtained by switching "ON/OFF" the two patch elements. The antenna can be used for narrowband applications such as the WLAN and WiMAX and wideband operations in the frequency range from 5 to $7 \mathrm{GHz}$ for other wireless standards. The design eliminates the need for using two ports as proposed in [17]-[18] and increases the number of possible frequency bands from one to four. The volume of the proposed antenna, including the ground plane and the substrate, is $50 \mathrm{~mm} \times 50 \mathrm{~mm} \times 1.57 \mathrm{~mm}$. The design can also be used for cognitive radio applications as described in [19]. 


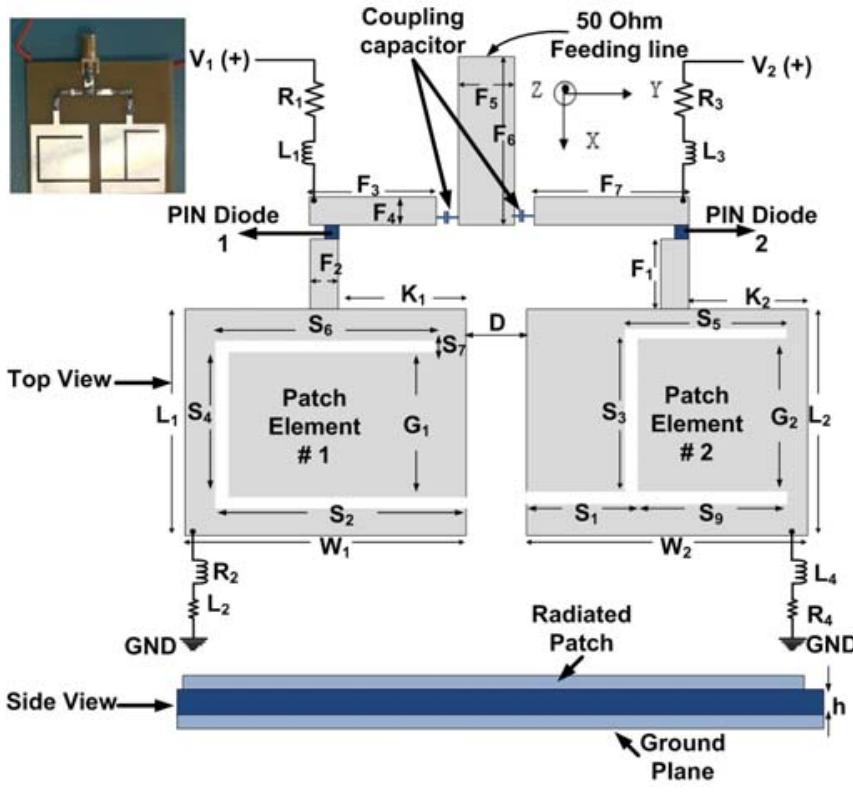

Fig. 1 Configuration of proposed antenna with DC biasing networks

\section{Antenna Configuration AND Design Procedure}

Figure 1(a) shows the schematic diagram of our proposed reconfigurable antenna which consists of two patch elements (patch elements \#1 and \#2) with a simple feed network, two PIN-diode switches and two chip capacitors all on one side of the substrate and a ground plane on the other side of the substrate. The complete antenna is designed using the EM simulator, HFSS V.11.2, based on Finite Elements Modeling (FEM) and fabricated on an FR-4 substrate with thickness of $1.57 \mathrm{~mm}$ and a relative permittivity of 4.4 . The key antenna parameters of the antenna are shown in Table I.

The dimensions of the patch elements of the antenna are computed to operate in the 5.5-GHz WLAN band using the equations given in [20]. Since cutting a slot on the radiator can change the current distribution and the current path, and hence improve the impedance matching especially at higher frequencies, as discussed in [21], in our design, we use two C-Slots on the two patch elements, as shown in Fig. 1(a), to generate a wide impedance bandwidth and to create multiple resonant frequencies. The feed network has a main $50-\Omega$ feed line and two connecting lines which have been optimized, in terms of impedance bandwidth, to have a line impedance of 63 $\Omega$. Two PIN diodes are placed on the connecting lines to the patch elements and used as switches. Just to prove our design concept, we have used the practical PIN diodes, SMP1320-079 from Skyworks Solutions Inc. with a size of $1.5 \times 0.7 \mathrm{~mm}^{2}$, as the switches. In computer simulation, these two diodes are modeled using the Resistance, Inductance and Capacitance (RLC) boundary sheet which gives $0.9 \Omega$ as the impedance value of the PIN diode in the ON state and $0.3 \mathrm{pF}$ as the capacitance value in the OFF state. These PIN diodes are turned "ON/OFF" using a DC biased signal, so two coupling chip capacitors $\left(\mathrm{C}_{1}\right.$ and $\left.\mathrm{C}_{2}\right)$ each with $10 \mathrm{pF}$ are used to prevent the
TABLE I: DETAILED DIMENSIONS OF PROPOSED ANTENNA (UNIT IN mm)

\begin{tabular}{|c|c|c|c|c|c|c|c|c|}
\hline $\mathbf{L}_{\mathbf{1}}$ & $\mathbf{W}_{\mathbf{1}}$ & $\mathbf{L}_{\mathbf{2}}$ & $\mathbf{W}_{\mathbf{2}}$ & $\mathbf{S}_{\mathbf{1}}$ & $\mathbf{S}_{\mathbf{2}}$ & $\mathbf{S}_{\mathbf{3}}$ & $\mathbf{S}_{\mathbf{4}}$ & $\mathbf{S}_{\mathbf{5}}$ \\
\hline 24 & 20 & 24 & 22 & 9 & 16 & 16 & 14 & 12 \\
\hline $\mathbf{S}_{\mathbf{6}}$ & $\mathbf{S}_{\mathbf{7}}$ & $\mathbf{S}_{\mathbf{9}}$ & $\mathbf{F}_{\mathbf{1}}$ & $\mathbf{F}_{\mathbf{2}}$ & $\mathbf{F}_{\mathbf{3}}$ & $\mathbf{F}_{\mathbf{4}}$ & $\mathbf{F}_{\mathbf{5}}$ & $\mathbf{F}_{\mathbf{6}}$ \\
\hline 16 & 1 & 11 & 9 & 2 & 9 & 2 & 3 & 11.6 \\
\hline $\mathbf{F}_{\mathbf{7}}$ & $\mathbf{D}$ & $\mathbf{K}_{\mathbf{1}}$ & $\mathbf{K}_{\mathbf{2}}$ & $\mathbf{G}_{\mathbf{1}}$ & $\mathbf{G}_{\mathbf{2}}$ & $\mathbf{h}$ & \multicolumn{2}{|c|}{ Ground PlaneArea } \\
\hline 12 & 4 & $\mathbf{9}$ & 11 & 14 & 16 & 1.57 & \multicolumn{2}{|c|}{$50 \times 50$} \\
\hline
\end{tabular}

DC signal from flowing to the main feed line but allow the RF current to pass through. The biasing networks for the two PIN diodes are also shown in Fig. 1, where the inductors $\mathrm{L}_{1}, \mathrm{~L}_{2}, \mathrm{~L}_{3}$ and $\mathrm{L}_{4}$, all with $12 \mathrm{nH}$, are used as radio-frequency (RF) chokes to provide high impedance for the RF signals. The resistors, $\mathrm{R}_{1}$, $R_{2}, R_{3}$ and $R_{4}$, each with $10 \mathrm{k} \Omega$, are used to control the DC biasing current to (or DC biasing voltage of about $0.7 \mathrm{~V}$ across) the PIN diodes. These lumped components will have insignificant effects on the antenna performance because the impedances of the RL circuits are much higher than the impedance of the antenna, allowing very little currents to flow through.

\section{SimUlation AND MEASUREMENTS RESUlTS}

\section{A. Impedance Bandwidth for $S_{11}<-10 d B$}

The two PIN diodes provide three possible and useful switching states, i.e., ON-OFF, OFF-ON and ON-ON states (note that the OFF-OFF state has no practical use). Simulation tests using the HFSS have been carried out on the impedance bandwidth (for reflection coefficient $\mathrm{S}_{11}<-10 \mathrm{~dB}$ ) of the antenna in different states. In the OFF-ON state, only patch element \#2 is $\mathrm{ON}$ and functioning. Simulation results in Fig. 2(a) show that a dual-band is obtained at 5.6 and $6.2 \mathrm{GHz}$, with the respective bandwidths of $5.2 \%$ and $4.85 \%$. In the ON-OFF state, only patch element $\# 1$ of the antenna is $\mathrm{ON}$ and radiating. The results in Fig. 2(b) show that another dual-band mode is obtained at 5 and $5.7 \mathrm{GHz}$, with the corresponding impedance bandwidth of $4.2 \%$ and $2.4 \%$. In the ON-ON state, both patch elements are radiating. A wide bandwidth of $33.52 \%$, covering the frequency range from 4.99 to $7 \mathrm{GHz}$, is obtained as shown in Fig. 2(c). To validate the simulation results, the proposed antenna has also been fabricated and the $\mathrm{S}_{11}$ in the ON-OFF, OFF-ON and ON-ON states have been measured using Agilent N5230A vector network analyzer. Results are shown in Figs. 2(a) - 2(c) for comparison. It can be seen that the simulated and measured results are in good agreement. The small discrepancies between the simulated and measured results could be attributed to the fabrication accuracy of the prototype.

\section{B. Effects of C-Slots}

Multiband operation of the antenna is achieved mainly by the C-Slots on the patch elements. Simulation tests have been carried out to study the $\mathrm{S}_{11}$ of the antenna without the C-Slots in the ON-OFF and OFF-ON states. Figure 3 compares the $S_{11}$ with and without the C-Slots in the patch elements. In the 


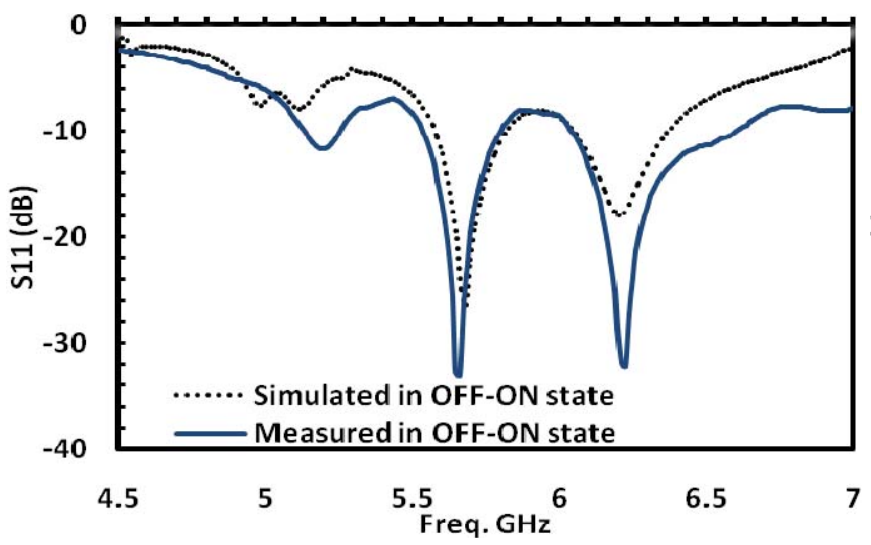

(a)

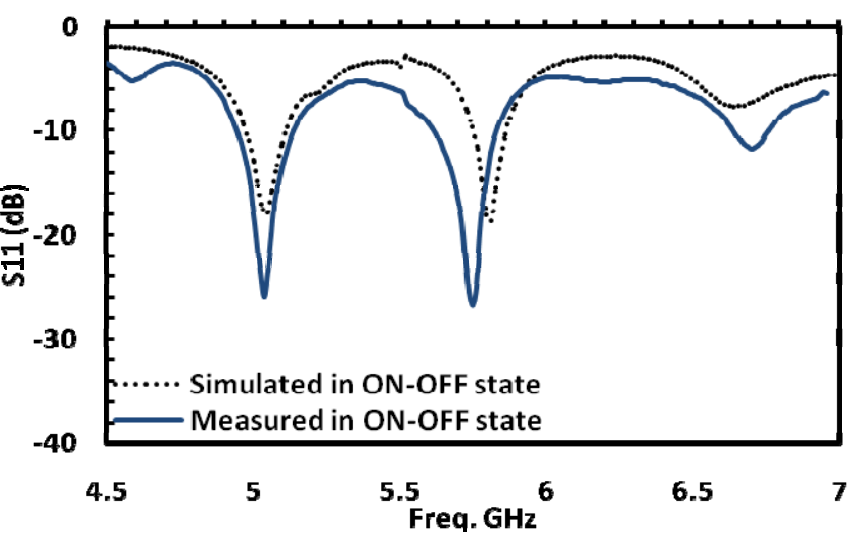

(b)

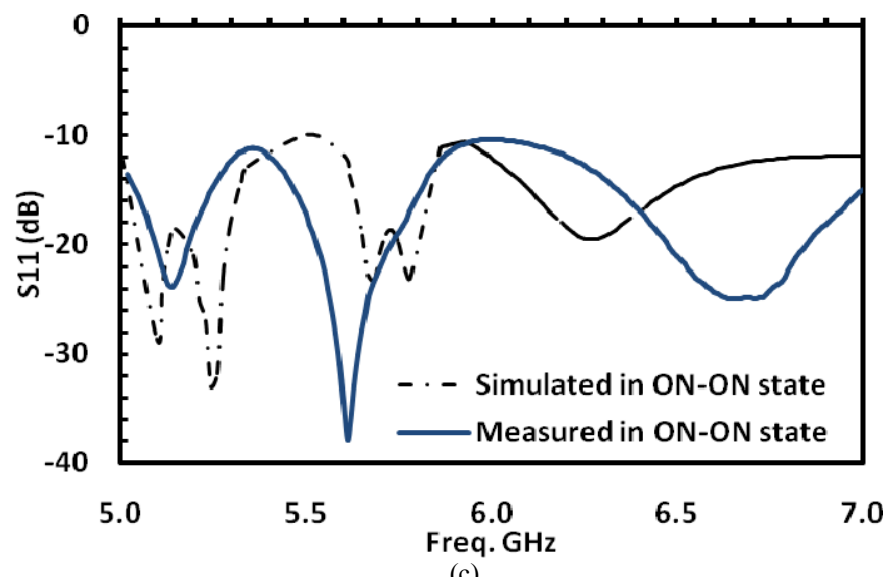

(c)

Fig. 2 Simulated and measured $\mathrm{S}_{11}$ of proposed antenna in (a) OFF-ON, (b) ON-OFF and (c) ON-ON states

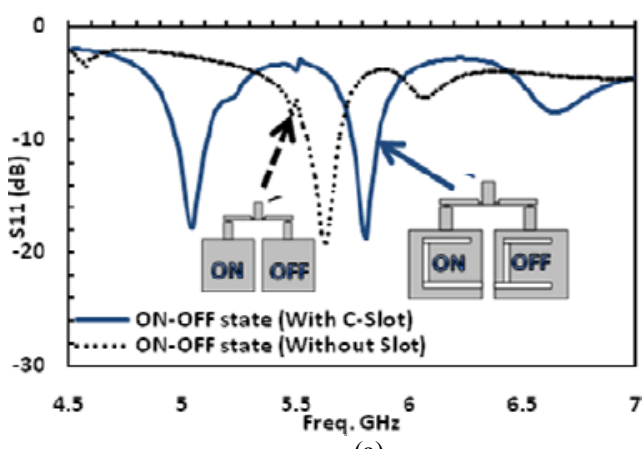

(a)

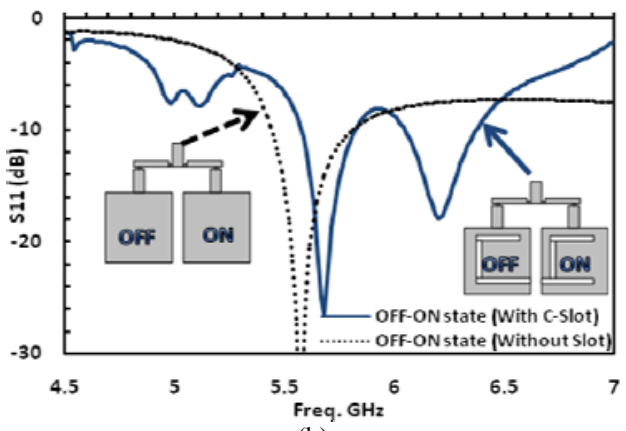

(b)

Fig.3 Effects of C-Slots on S11 in (a) ON-OFF and (b) OFF-ON states

ON-OFF state when patch element $\# 1$ is active and patch element \#2 is OFF, Fig. 3(a) shows that, without the C-Slots, the antenna has a resonant frequency at $5.6 \mathrm{GHz}$. While with the C-Slots, a dual-band is generated at 5 and $5.7 \mathrm{GHz}$. In the OFF-ON state when patch element \#2 is turned $\mathrm{ON}$ and patch element \#2 is OFF, Fig. 3(b) shows that the antenna without the $\mathrm{C}$-Slots has a single band at $5.55 \mathrm{GHz}$. While with the C-Slots on the patch elements, a dual-band is generated at 5.6 and 6.2 GHz. Therefore, the C-Slots on the patch elements help generate a dual band. Moreover, simulation results have also shown that the widths and the lengths of the patch elements determine the centre frequencies in the single-band cases. While the positions and dimensions of the C-Slots on the patch elements determine the centre frequencies in the dual-band cases. The simulated-frequency bands generated with and without the C-Slots in our design are summarised in Table II.

TABLE II GENERATED BANDS WITH AND WITHOUT C-SLOTS

\begin{tabular}{|c|c|c|}
\hline State & Without Slots & With C-Slots \\
\hline OFF ON & $5.55 \mathrm{GHz}$ & $5.6 \& 6.2 \mathrm{GHz}$ \\
\hline ON OFF & $5.6 \mathrm{GHz}$ & $5 \& 5.7 \mathrm{GHz}$ \\
\hline ON ON & $5.55 \& 5.65 \mathrm{GHz}$ & Wideband from 5 to $7 \mathrm{GHz}$ \\
\hline
\end{tabular}




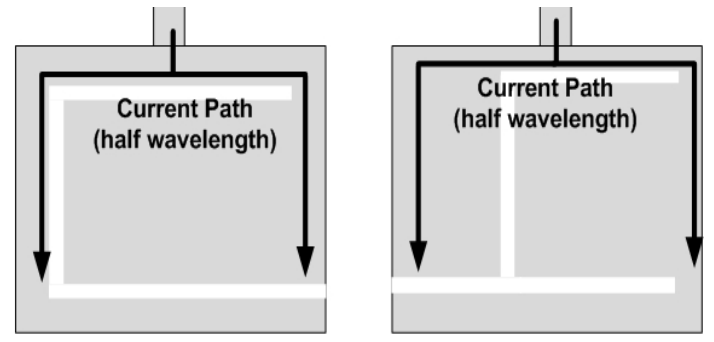

Fig.4 Current paths on patch elements with slots

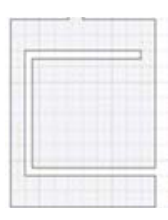

$5.6 \mathrm{GHz}$

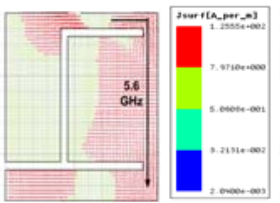

(a) OFF ON

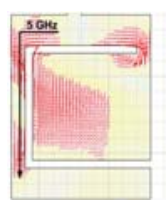

$5 \mathrm{GHz}$

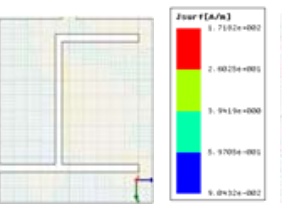

(b) ON OFF

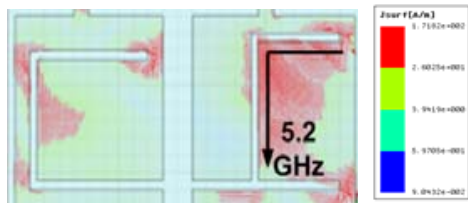

$5.2 \mathrm{GHz}$

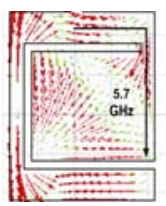

$5.7 \mathrm{GHz}$

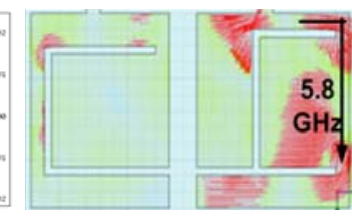

$5.8 \mathrm{GHz}$

(c) ON ON

Fig. 5 Simulated current distributions in (a) OFF-ON, (b) ON-OFF and (c) ON-ON states

\section{Current Distributions}

Cutting slots on the radiator of an antenna can change the current path and so can be used to generate dual- or multiple-bands operation. If the slots are absent in our proposed design, the antenna will have only one major current path on each of the patch elements, with the current-path length corresponding to $\lambda / 2$ of the resonant frequency. However, in the presence of the two C-slots, the current path on each of the patch elements is separated into 2 paths as shown in Fig. 4, hence creating dual-band operation. The lengths of individual current path on each of the patch elements correspond to $\lambda / 2$ of the resonant centre frequencies of the respective dual bands. Figure 5 shows the simulated current distributions on the patch elements in different switching states. In the OFF-ON state, patch element $\# 2$ is radiating and patch element $\# 1$ is OFF.
Figure 5(a) shows that the current travels around the C-Slot on patch element $\# 2$, as expected, generating the resonant frequencies at 5.6 and $6.2 \mathrm{GHz}$ as shown in Fig. 2(a) for the WLAN $802.11 \mathrm{a} / \mathrm{h} / \mathrm{j} / \mathrm{n}$ applications. In the ON-OFF state, only patch element \#1 is $\mathrm{ON}$ and radiating and patch element $\# 2$ is OFF. Figure 5(b) shows that the current travels around the C-Slot on patch element \#1, generating the dual band at 5 and 5.7 GHz as shown in Fig. 2(b), for the WLAN 802.11 $\mathrm{a} / \mathrm{h} / \mathrm{j} / \mathrm{n}$ applications and WLAN $802.11 \mathrm{~B}$ and $\mathrm{G}$ applications, respectively. In the ON-ON state where both patch elements are ON, Fig. 5(c) shows the current paths corresponding to $\lambda / 2$ of the resonant frequencies at 5.2 and $5.8 \mathrm{GHz}$ as shown in Fig. 2(c). The wideband operation is obtained by the coupling which will be explained later.

\section{Measured Radiation Patterns and Gain}

The radiation patterns of the antenna have been measured using the Small Antenna Radiated Testing Range (SMART) at the National Physical Laboratory (NPL), with results normalized to the maximum values. Figures 6 to 8 show the measured and simulated co- and cross-polarization patterns of the antenna in different switch states at several frequencies across the operating bandwidth.

In the OFF-ON and ON-OFF states, the radiation patterns at the pair-resonant frequencies of 5.6 and $6.2 \mathrm{GHz}$, and 5 and 5.7 $\mathrm{GHz}$, respectively, are used for comparison. In the ON-ON state where the antenna has an operation bandwidth from 5 to 7 $\mathrm{GHz}$, the radiation patterns at the extreme frequencies of 5 and $7 \mathrm{GHz}$ and the middle frequency of $6 \mathrm{GHz}$ are studied. From the current distributions shown in Fig. 5, it can be seen that the directions of the dominant current paths at the frequencies studied are mainly in the $\mathrm{X}$-direction, so the $\mathrm{Y}-\mathrm{Z}$ and $\mathrm{X}-\mathrm{Z}$ planes are the $\mathrm{H}$ - and E-planes, respectively. As a result, the co-polarization patterns in Figs. 6-8 are all relatively unidirectional toward the Z-direction with small back radiation due to the finite ground-plane size. No major change can be found in the radiation patterns in the three switch states of OFF-ON, ON-OFF and ON-ON. Some minor discrepancies occur between the simulated and measured results, which could be due to the effect of the coaxial cable connected to the antenna during measurements.

The peak gains of the antenna in different states have also been observed and results have shown that no significant change in the peak gains. The peak gains at different frequencies are between 3 and $5 \mathrm{dBi}$ in different switching states and summarized in Table III. The simulated radiation efficiency of the antenna in the ON-ON state ranges from $60 \%$ to $70 \%$.

\begin{tabular}{|c|c|c|c|}
\hline $\begin{array}{c}\text { TABLE III: PEAK GAINS } \\
\text { frequency bands (GHz) }\end{array}$ & $\begin{array}{c}\text { ON OFF } \\
\mathbf{( 5 ~ \& ~ 5 . 7 )}\end{array}$ & $\begin{array}{c}\text { OFF ON } \\
\mathbf{( 5 . 6} \& \mathbf{6 . 2})\end{array}$ & $\begin{array}{c}\text { ON ON } \\
\mathbf{( 5 ~ \& ~ 7 )}\end{array}$ \\
\hline Gain in dBi & 3.7 and 3.54 & $3.6 \& 4.2$ & $3.17 \& 4.92$ \\
\hline
\end{tabular}

\section{INDEPENDENT CONTROL OF EACH BAND THROUGH C-SLOTS}

To design antennas with multiple-band operations, it is desirable to have independent-frequency controls on the 


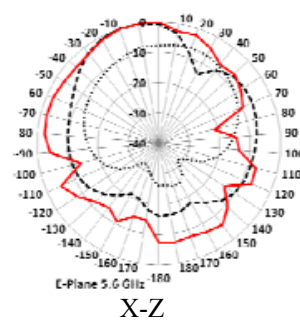

(a)
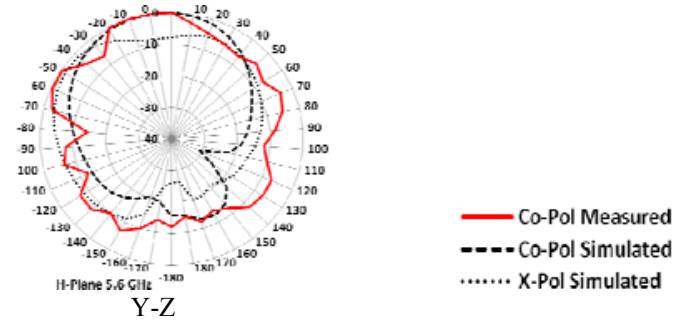

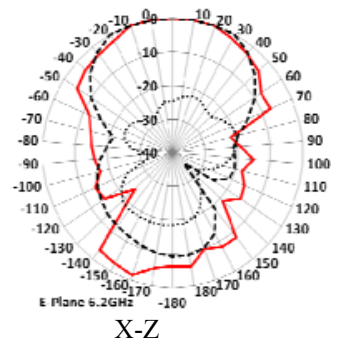

(b)

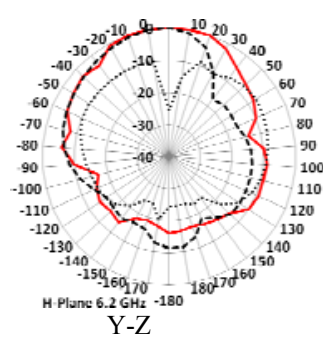

Fig. 6 Simulated and measured Co and X-pol in E and H-planes in OFF-ON state at (a) $5.6 \mathrm{GHz}$ and (b) $6.2 \mathrm{GHz}$

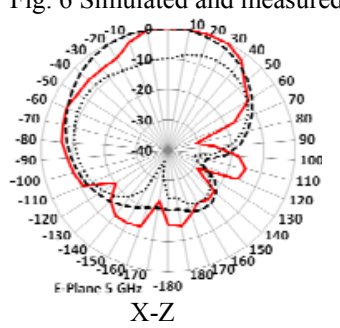

(a)
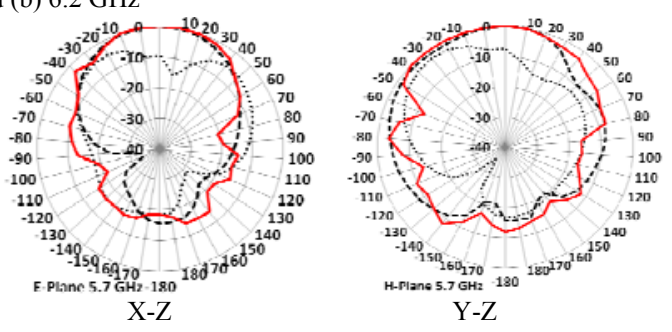

(b)

Fig. 7 Simulated and measured Co and X-pol in E and H-planes in ON-OFF state at (a) $5 \mathrm{GHz}$ and (b) $5.7 \mathrm{GHz}$

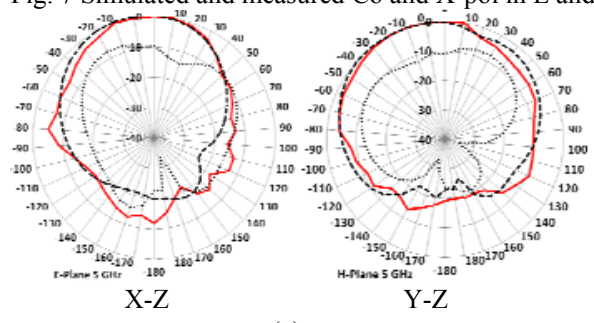

(a)

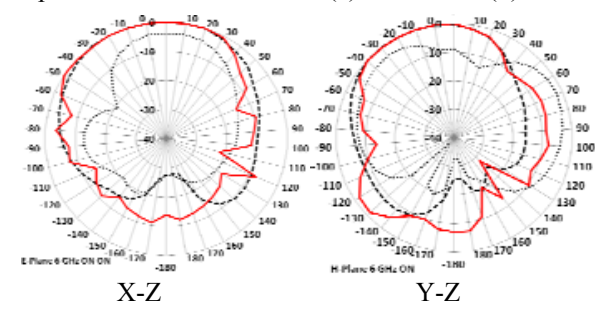

(b)

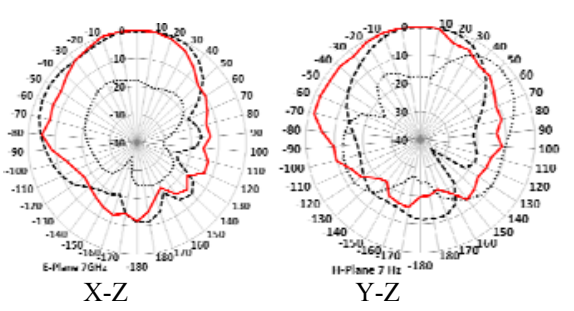

(c)

Fig. 8 Simulated and measured Co and X-pol in E and H-planes in ON-ON state at (a) $5 \mathrm{GHz}$, (b) $6 \mathrm{GHz}$ and (c) 7 GHz.

frequencies. Achieving this option is very challenging. Very often, when one parameter is changed, all the frequencies are affected [22]-[23] and the antenna needs to be completely re-optimized. Sometimes, the shape of the resigned antenna has to be significantly changed, causing a lot of inconvenience in designing wireless devices.

Results in previous sections have shown that, in the ON-OFF and OFF-ON states, the C-Slots on the patch elements of the antenna can be used to generate two frequency bands for dual-band operation. Here we show how to use the C-Slots to independently control the frequency bands for dual-band operation without affecting the wideband operation. In the ON-OFF state, the simulation results in Fig. 9(a) shows the effects of changing the length $S_{4}$ in the slot of patch element \#1 on the lower band of the dual band. It can be seen that increasing $\mathrm{S}_{4}$ moves the 5-GHz band lower but keeps the 5.7-GHz band fixed. Figure 9(b) shows that the effect of changing $\mathrm{W}_{1}$ and $\mathrm{S}_{2}$ together on the higher band of the dual band. Here, reducing $\mathrm{W}_{1}$ and $\mathrm{S}_{2}$ together moves the $5.7-\mathrm{GHz}$ band to a higher frequency band, yet the 5-GHz band remains unchanged. In the OFF-ON state, Fig. 10(a) shows the effects of changing the position of the whole C-Slot up and down on patch element \#2, indicating that the position of the C-Slot can be used to move the lower band (at around $5.6 \mathrm{GHz}$ ), yet keeping the $6.2-\mathrm{GHz}$ band fixed. Finally, Fig. 9(b) shows that changing the distance $\mathrm{G}_{2}$ (i.e., moving $\mathrm{S}_{1} \& \mathrm{~S}_{9}$ together closer or further from $\mathrm{S}_{5}$ while keeping $\mathrm{S}_{3}$ the same) can shift the higher band (at around $6.2 \mathrm{GHz}$ ) to a higher or lower frequency band, yet maintaining the 5.6-GHz band. It should be noted that, in some cases, after moving the frequency of the band, we may need to optimize the other parameters of the antenna to achieve the desirable $\mathrm{S}_{11}$. These results show that we can independently control the frequencies of the dual bands by using the C-Slots on the patch elements. Simulation results have also shown that the wideband performance in the ON-ON state is not affected when these narrow bands are moved to other frequencies. This degree of freedom further enhances the antenna capability. More simulation tests have shown that the maximum frequency separations that can be achieved between the dual-band in the ON-OFF and OFF-ON states are $130 \mathrm{MHz}(5.45-5.58 \mathrm{GHz})$ and $290 \mathrm{MHz}(5.8-6.09 \mathrm{GHz})$, respectively.

Based on the above discussions, we propose the following simple guidelines to design an antenna for dual-band operation at desirable frequencies:

1. Use the equations given in [20] to compute the dimensions of the patch elements to operate at the desirable frequency band, e.g. WLAN band.

2. Set the dimensions and positions of the C-Slots to our proposed antenna to start with.

3. Adjust the locations and dimensions of the C-Slots on each patch elements to achieve the desirable dual bands. Since they can be independently controlled, this is not difficult to do. 


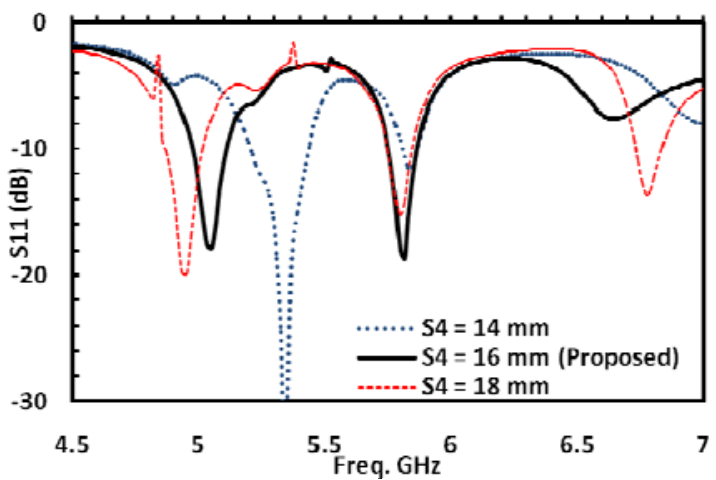

(a)

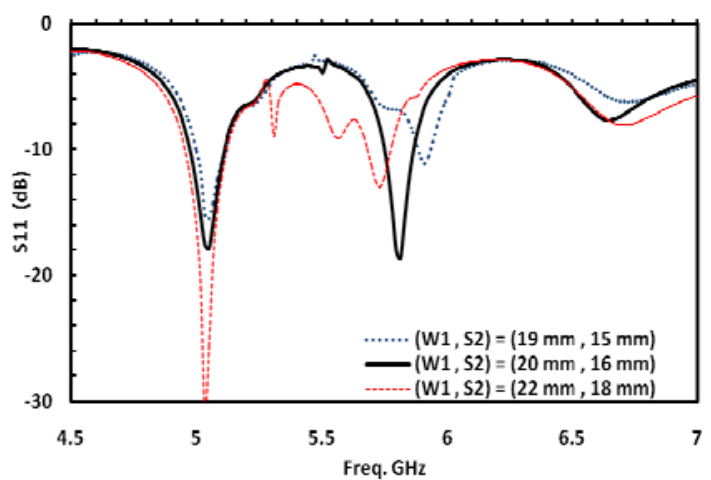

(b)

Fig. 9 In ON-OFF state: (a) effects of $\mathrm{S}_{4}$ on lower band of dual band and (b) effects of $\mathrm{W}_{1}$ and $\mathrm{S}_{2}$ together on higher band of dual band

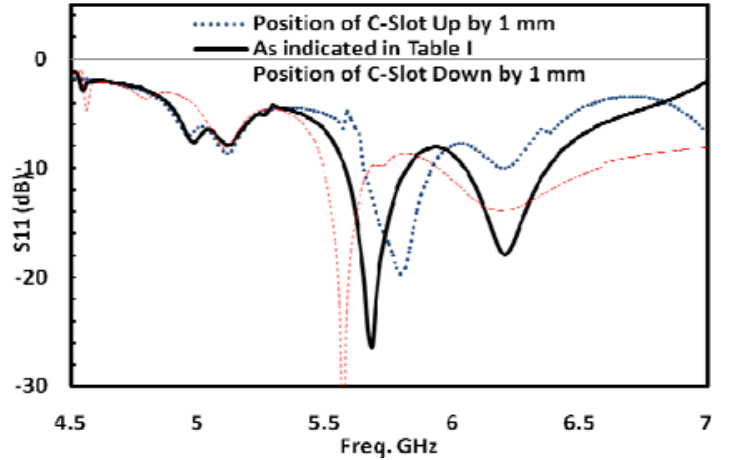

(a)

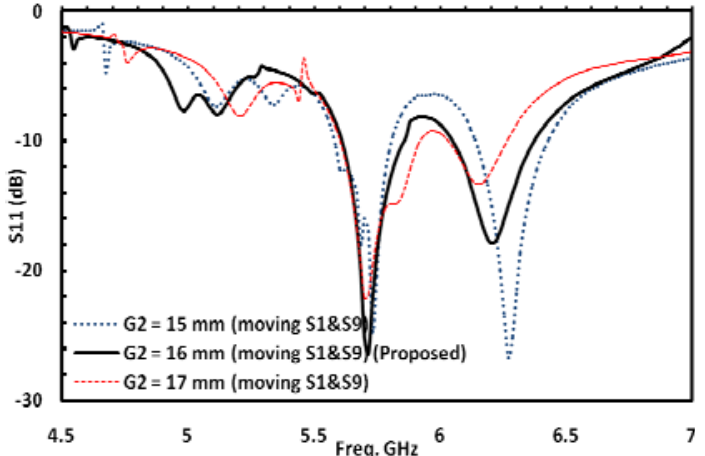

(b)

Fig. 10 In OFF-ON state, (a) effects of C-Slot position on lower band of dual band and (b) effects of $\mathrm{G}_{2}$ on higher band of dual band.

4. Optimize the dimensions of the feed network to the patch elements, which is essential for wideband operation.

5. Optimize the locations of the switches and coupling capacitors, which are very sensitive to the antenna $S_{11}$.

\section{EFFECTS OF COUPLING}

Previous results have shown that when both patch elements are $\mathrm{ON}$, the antenna has a wide bandwidth. This must be the results of mutual coupling between the patch elements, which is examined here. To study the coupling effects between the 2 patch elements, we place an EM wave absorber (high lost material) between the 2 patch elements in the simulation model as shown in Figs. 11 (a)-(b) to remove the coupling effects and simulated the impedance bandwidth using $\mathrm{S}_{11}$ in the ON-OFF, OFF-ON and ON-ON states.

With patch element \#1 turned ON, patch element \#2 turned $\mathrm{OFF}$, and the coupling effect from element \#2 to element \#1 removed by the absorber, the simulated $S_{11}$ is shown in Fig. 12(a). For comparison, the simulated $S_{11}$ without the absorber, i.e. with coupling, is also shown in the same figure. It can be seen that the differences in $S_{11}$, particularly near the dual frequency bands, are quite insignificant, indicating that the coupling between the two patch elements is very small. With patch element \#1 turned OFF and patch element \#2 turned ON, the simulated $\mathrm{S}_{11}$ with and without the absorber are shown in Fig. 12(b). The differences in $S_{11}$ near the dual-frequency bands are slightly noticeable. Nevertheless, it is insignificant and so the coupling between the two patch elements is still very small. With both patch elements \#1 and \#2 are ON, i.e. in the ON-ON state, the simulated $\mathrm{S}_{11}$ with and without the absorber are shown in Fig. 12(c). It can be seen that the mutual coupling between the 2 elements is much stronger and significantly reduces the $S_{11}$ to less than $-10 \mathrm{~dB}$ across the whole frequency band. As a result, the operation bandwidth of the antenna is much wider.

\section{CONCLUSIONS}

A reconfigurable multiband and wideband patch antenna, employing dual-patch elements and C-Slots with a compact volume of $50 \times 50 \times 1.57 \mathrm{~mm}^{3}$, has been presented and studied using simulation and measurement. Two PIN diode switches are used to switch $\mathrm{ON}$ and OFF two patch elements to operate the antenna in two different dual-band modes or a wideband mode (with a bandwidth of $33.52 \%$ ). The frequencies in the dual-band modes can be independently control using the C-Slots without affecting the wideband performance. Simulation and calculation results have shown that the wideband performance is achieved by the coupling effects between the patch elements. The measured and simulated results have shown that radiation patterns across $5-7 \mathrm{GHz}$ are stable in different modes. The main advantages of the proposed antenna include low profile, lightweight and easy to fabricate 


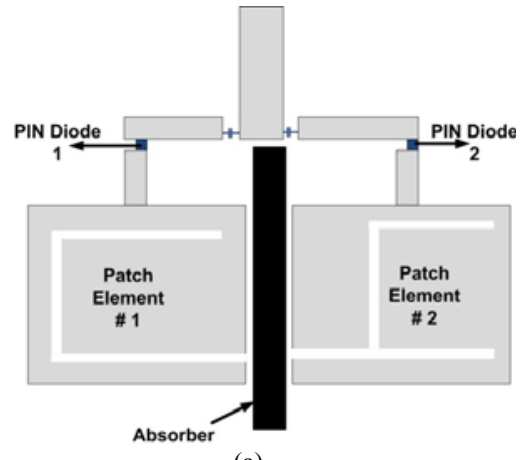

(a)

Fig. 11 Antenna with absorber to remove coupling effects (a) Top view and (b) side view

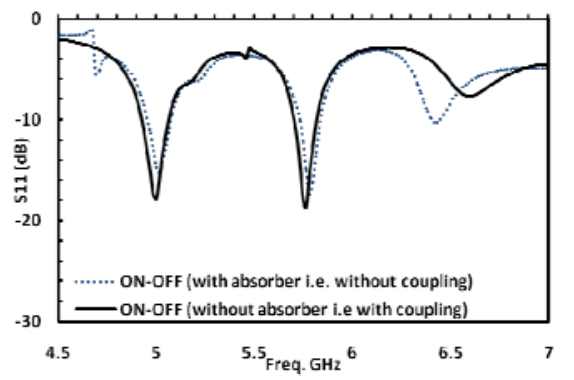

(a)

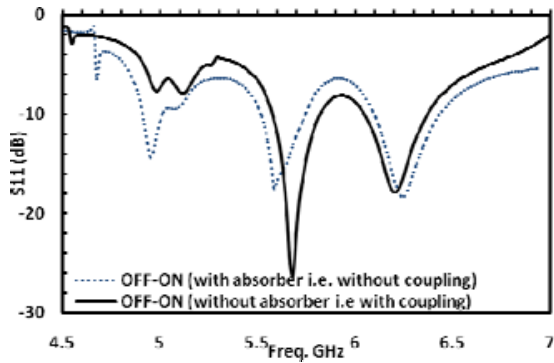

(b)

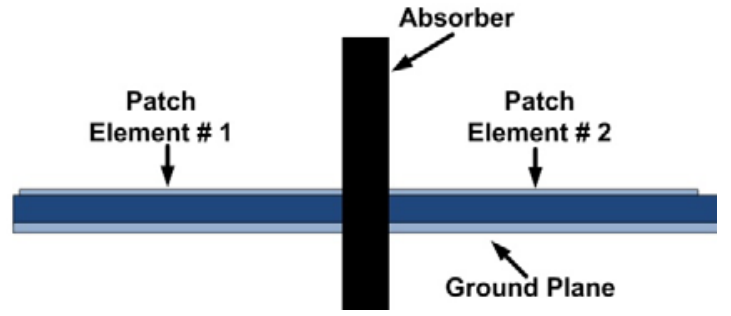

(b)

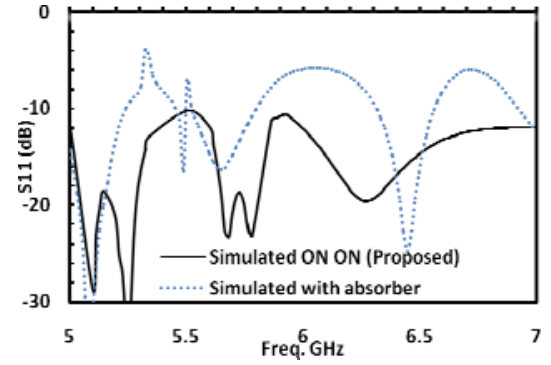

(c)

Fig. 12 Reflection coefficient $\left(\mathrm{S}_{11}\right)$ with absorber in (a) ON-OFF (b) OFF-ON and (c) ON-ON states

simple structure targeting future smaller wireless communication devices.

\section{Acknowledgments}

The measurements at the NPL SMART chamber were supported by the Measurements for Innovators (MFI) program and the National Measurement Office, an Executive Agency of the Department for Business, Innovation and Skills. The authors would like to acknowledge the Skyworks Solutions Inc. company for providing samples used in this work.

\section{REFERENCES}

[1] G. Kumar and K.P Ray, "Broadband Microstrip Antennas", Artech House, Boston, MA and London, 2003, pp. 18-23.

[2] D.M Pozar and D.H. Schaubert "Microstrip Antennas", New York 1995, IEEE press.

[3] H. Wang, X. B. Huang and D. G. Fang, "A Single Layer Wideband U-Slot Microstrip Patch Antenna Array", IEEE Antennas and Wireless Propagation Letters, vol. 7, pp. 9-12, 2008.

[4] C.L. Mak, R. Chair, K.F. Lee, K.M. Luk and A.A. Kishk, "Half U-slot patch antenna with shorting wall", Electronics Letters, vol. 39, pp. 1779-1780, 2003.

[5] Y. Li, R. Chair, K.M. Luk and K.F. Lee, "Broadband triangular patch antenna with a folded shorting wall," IEEE Antennas and Wireless Propagation Letters, vol. 3, pp. 189-192, 2004.

[6] S. Qu and Q. Xue, "A Y-Shaped Stub Proximity Coupled V-Slot Microstrip Patch Antenna", IEEE Antennas and Wireless Propagation Letters, vol. 6, pp. 40-42, 2007.

[7] Y. Lee and J. Sun, "A New Printed Antenna for Multiband Wireless Applications", Antennas and Wireless Propagation Letters, IEEE, vol. 8, pp. 402-405, 2009.

[8] J. Anguera, C. Puente, C. Borja and J. Soler, "Dual-Frequency Broadband-Stacked Microstrip Antenna Using a Reactive Loading and a Fractal-Shaped Radiating Edge", IEEE Antennas and Wireless Propagation Letters, vol. 6, pp. 309-312, 2007.

[9] K-L. Wong and W-H. Hsu, "A Broad-Band Rectangular Patch Antenna with a Pair of Wide Slits," IEEE Transaction Antennas Propagation, vol. 49, no. 9, pp. 1345-1347, Sept. 2001
[10] F. Yang, X. Zhang, X. Ye and Y. Rahmat-Samii,“ Wide-Band E-Shaped Patch Antennas for Wireless Communications," IEEE Transaction Antennas Propagation, vol. 49, no. 7, pp. 1094-1100, July. 2001.

[11] R. Bhalla and L. Shafai, "Broadband patch antenna with a circular arc shaped slot," IEEE Antennas and Propagation Society International Symposium, 2002. vol.1, no., pp. 394- 397 vol.1, 2002

[12] A. Sheta and S.F. Mahmoud, "A Widely Tunable Compact Patch Antenna", IEEE Antennas and Wireless Propagation Letters, vol. 7, pp. $40-42$

[13] A.C.K. Mak, C.R. Rowell, R.D. Murch and C-L Mak, "Reconfigurable Multiband Antenna Designs for Wireless Communication Devices", IEEE Transactions on Antennas and Propagation, vol. 55, pp. 1919-1928, 2007

[14] S. Yang, C. Zhang, H. Pan, A. Fathy and V. Nair, "Frequency-reconfigurable antennas for multiradio wireless platforms", IEEE Microwave Magazine, vol. 10, pp. 66-83, 2009.

[15] Y. Huang and K. Boyle, Antennas: From Theory to Practice. Hoboken, NJ: Wiley, 2008. Ch. 8.

[16] E. Ebrahimi and P.S. Hall, "A dual port wide-narrowband antenna for cognitive radio", 3rd European Conference on Antennas and Propagation, EuCAP 2009. pp. 809-812, 2009.

[17] F. Ghanem, P.S. Hall and J.R. Kelly, "Two port frequency reconfigurable antenna for cognitive radios", Electronics Letters, vol. 45, pp. 534-536, 2009.

[18] R. Kelly, P.S. Hall and P. Gardner, "Integrated wide-narrow band antenna for switched operation", 3rd European Conference on Antennas and Propagation, EuCAP 2009, pp. 3757-3760, 2009.

[19] H. F. AbuTarboush, S. Khan, R. Nilavalan, H. S. Al-Raweshidy and D. Budimir, "Reconfigurable wideband patch antenna for cognitive radio", Loughborough Antennas \& Propagation Conference, LAPC 2009, pp. 141-144, 2009.

[20] C.A. Balanis, Antenna Theory, 2nd ed. New York: John Wiley \& Sons, Inc., 1997.

[21] J. Volakis, "Antenna Engineering Handbook," McGraw Hill, 2007. Ch. 16.

[22] R. Sujith, V. Deepu, D. Laila, C. Aanandan, K. Vasudevan and P. Mohanan, "A compact dual-band modified T-shaped CPW-fed monopole antenna", Microwave and Optical Technology Letters, vol. 51, no. 4, pp. 937-939, 2009

[23] S. Lee, H. Park, S. Hong and J. Choi, "Design of a Multiband Antenna using a Planner Inverted-F Structure," The 9th International Conference on Advanced Communication Technology, vol. 3, pp.1665-1668, Feb. 2007. 
